\title{
The Usefulness of Biosynthetic Vascular Graft Omniflow II and Autologous Veins for the Treatment of Massive Infection of Dacron Vascular Graft with Enterococcus faecalis HLAR
}

\author{
WITOLD WOŹNIAK*, ROBERT BAJNO, MICHAŁ ŚWIDER and PIOTR CIOSTEK
}

First Department of General and Vascular Surgery, Second Faculty of Medicine with the English Division and the Physiotherapy Division, Medical University of Warsaw, Warsaw, Poland

Submitted 12 July 2016 and accepted 13 September 2016

\begin{abstract}
Infections of vascular grafts are the most severe complications in vascular surgery. We present the case of a 73-year-old male with infection of a dacron prosthesis with a strain of Enterococcus faecalis. The patient was treated with replacement of a full prosthesis with a combined graft constructed from Biosynthetic Vascular graft Omniflow and autologous veins. This graft is recommended for implantation in patients with a higher risk of infection. Our case is one of the first reported usage of this kind of graft in the aortic region and in a 2 years observation period no recurrence of infection was observed.
\end{abstract}

Ke y w ord s: Enterococcus faecalis, abdominal aortic surgery, Omniflow II, vascular graft infection, vascular prosthesis

Infections of synthetic vascular grafts are undoubtedly the most severe complications in vascular surgery and despite the continuous advancement of the medical science, the results of treatment of such complications have not improved. The percentage of amputations and deaths among these patients continues to remain at a high level, reaching 20-30\% (Samson et al., 1988). The risk of an infection of a graft in aorto-femoral section has been described as $0.5-3 \%$ (Swain et al., 2004) and it is extremely closely related to the place of implantation. The likelihood of a graft infection is moreover increased by a number of factors such as extended time of an operation, the presence of different catheters and drains, intra- and postoperative chyle leak, coexisting infections, immunodeficiency conditions, advanced age, malnourishment, diabetes mellitus or chronic immunosuppression. The lack of observance to the principles of aseptic techniques also has an impact.

The most common etiological factors are Staphylococcus strains which are responsible for more than $53 \%$ of all aorto-femoral infections. They produce glycocalyx, which on the one hand allows adhesion to the graft surface and on the other constitutes a component of biofilm providing protection against phagocytosis, antibodies and antibiotics (Bandyk et al., 1991). Grampositive cocci of Enterococcus sp. cause an infection to a much lesser extent in grafts in the aorto-femoral location. Several decades ago they were considered relatively pathogenic - little virulence and minor percentage of infections combined with their common occurrence in a human environment. The wide implementation of cephalosporins, to which Enterocci has a natural resistance, has led to their domination in gastrointestinal microbiota, which resulted in a higher rate of severe infections caused by these bacteria among hospitalized patients. Moreover, long-term application of a combination of penicillin and high concentrations of aminoglycosides in treatment of endocarditis caused by enterococci has led to the formation of HLAR strains. In turn, the extensive use of vancomycin in hospital therapy (for prevention and treatment of MRSA infections) with time resulted in the evolution of VRE strains (with varying degrees of resistance to vancomycin and teicoplanin). Of course, there are other determinants that are responsible for the pathogenicity of enterococci, including the ability to produce cytolysin (hemolysin), gelatinases, a serine protease, collagen binding protein (Ace) and fibrinogen (SagA), aggregation substance (AS), the extracellular surface protein (Esp), the cell wall adhesin (protein Efa), peroxides, hyaluronidase, lipoteichoic acid, DNase, lipase and polysaccharide capsule. The presence of these factors results in

\footnotetext{
* Corresponding author: W. Woźniak, First Department of General and Vascular Surgery, Medical University of Warsaw, Warsaw, Poland; e-mail: drww@onet.pl
} 


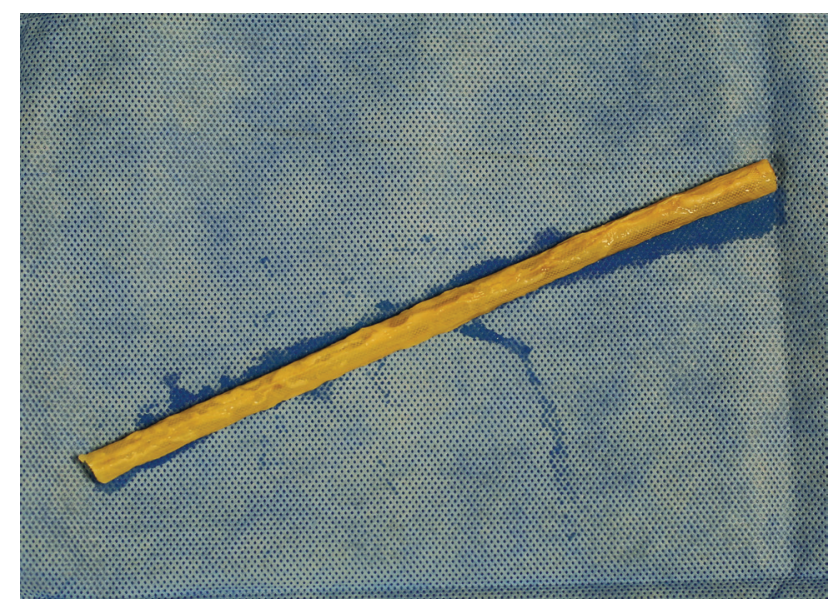

Fig. 1. Biosynthetic vascular graft Omniflow II

the ability of adhesion, colonization of the graft's surface and formation of protective biofilm. The dominant species among enterococci causing these infections are strains of Enterococcus faecalis, which comprise 70-80\% of the strains (Bronk and Samet, 2008).

A major dilemma faced by a surgeon in view of a vascular graft infection is the selection of treatment. Currently it is commonly agreed that the removal of the infected graft and a concurrent vascular reconstruction is the best method. While surgical removal of the infected graft does not raise concerns in most cases, the reconstruction stage is associated with the necessity of selecting the optimal location of the reconstructed vascular bed (extra-anatomical bypass or in situ bypass graft) together with the material necessary for its formation (autologous vessels, cadaver homograft, silvercoated grafts or antibiotic-soaked vascular grafts).

The authors also encountered the problems presented above based on the case of a 73-year-old patient with a massive infection of a dacron aortobifemoral vascular graft. The patient was initially operated on due to symptomatic Leriche syndrome with ischemia of both lower extremities stage $\mathrm{III}^{\circ}$, according to the Fontaine's scale (20-30 m intermittent claudication, rest pain, especially severe at night). The patient received a $18 / 9 \mathrm{~mm}$ bifurcated dacron graft. The upper anastomosis was performed with the aorta below the origin of the inferior mesenteric artery and the lower fixations on both sides over femoral arteries bifurcation. In the early postoperative period (day 8), a massive leak of serosanguineous fluid appeared from the wound located in the right groin, and afterwards also from the left groin. During the next days, the content leaking from the wound gradually changed its character to purulent. Throughout the whole postoperative period the patient did not present any fever and inflammatory markers were not specific for massive infection (leukocytosis 7000-9500/ $\mathrm{mm}^{3}$, CRP 33-37 mg\%).

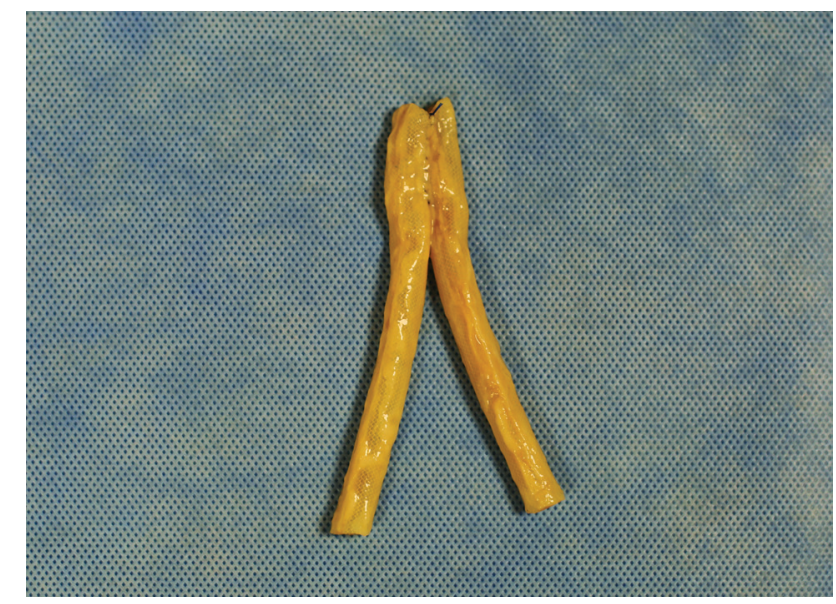

Fig. 2. Bifurcated prosthesis created by connecting two arms Omniflow II

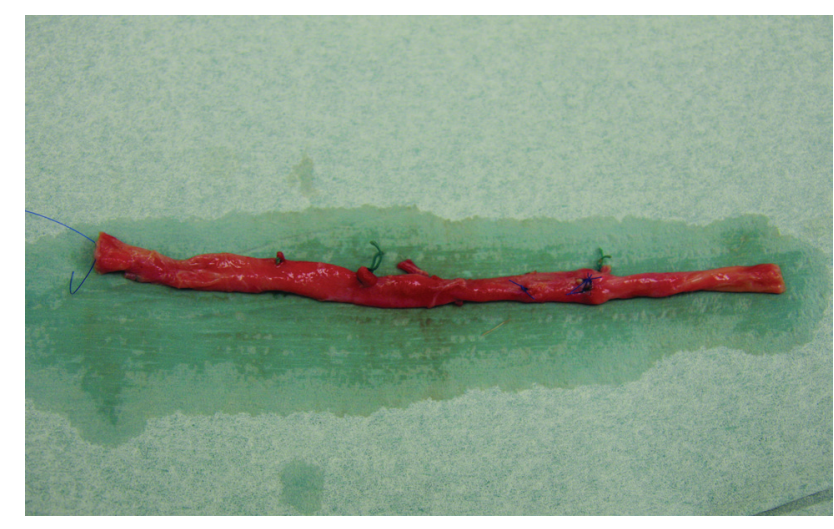

Fig. 3. One of two femoral veins harvested from the patient

Microbiological examination of the wound in left groin detected E. faecalis HLAR. Abdominal and Pelvic Computed Tomography revealed the presence of inflammatory infiltrate around both branches of the graft and an abscess in the distal anastomosis area on the right side, with no evident signs of infection within the aortic segment of the graft. It was decided that the patient should be reoperated. During the operation a total lack of the ingrowth healing process of the primary graft and features of infection (purulent fluid around the graft), also in the vicinity of the proximal anastomosis to the aorta, were noticed. Dacron prosthesis was entirely removed and replaced by a combined aortobifemoral graft constructed from biosynthetic vascular graft Omniflow II and two femoral veins harvested from the patient (Fig. 1-3). To this end a straight section of the Omniflow II graft ( $8 \mathrm{~mm}$ in diameter and $20 \mathrm{~cm}$ in length) was cut intraoperatively into two equal parts and the walls were incised longitudinally $(2-2,5 \mathrm{~cm}$ section). Afterwards the two parts were anastomosed with each other (side to side) in the previously mentioned incisions to give a bifurcated prosthesis with size $16 / 8 \mathrm{~mm}$. Then, reversed 18/19-cm-long segments of both femoral veins obtained from the patient were sutured to the 
branches of the graft (end-to-end). The combined biosynthetic-autologous prosthesis constructed that way was implanted in place of the removed aortobifemoral graft. In the first hours after surgery, acute ischemia of the left lower limb occurred in the course of thrombosis of the left branch of implanted bypass, which demanded surgical thrombectomy. Further hospitalization proceeded without any significant complications and the patient was discharged from the hospital. It should be noted that throughout the entire period of the hospital treatment a constant antibiotic therapy was used, despite obtaining negative cultures from the fluid surrounding the aortic section of the removed graft (however pathogens such as E. faecalis, which are capable of creating bacterial biofilm coating implanted materials are very rarely detected in the inflammatory exudate). Control Computed Tomography Angiography was performed 3 months after reoperation, which indicated complete regression of the inflammatory infiltrate described in the previous test and the presence of significant stenosis ( $80 \%$ on the right side and $85 \%$ on the left side) in anastomoses of the Omniflow II prosthesis with autological femoral veins. Due to the progressive symptoms of ischemia of both lower limbs related to the gradual progression of these lesions, the patient was qualified for a planned endovascular procedure. An effective angioplasty of both anastomoses of bioprosthesis with femoral veins was performed, complemented by bilateral implantation of stents. Satisfactory hemodynamic effect and complete regression of reported symptoms were achieved. In the nearly 2 -year ambulatory follow-up the patient did not show any signs of recurrence of infection within the vascular system or blood circulation disorders of the lower limbs.

In spite of conviction of the need for complete removal of a vascular graft in case of infection, attempts of conservative treatment (antibiotic therapy) or surgery limited to the direct focus of infection are still reported. This results from the often-isolated nature of an infection and the relatively high risk of complications that may occur during the removal of a prosthesis and reconstruction of a vascular bed. The scope of these operations may involve the removal of necrotic tissues, evacuation of inflammatory fluid reservoirs, irrigation drainage, local antibiotic therapy, coverage of bare sections of prostheses with well-vascularized muscle lobes and in selected cases segmental resection of the infected part of the graft with the reconstruction of the defect. In the described case the infection involved the entire graft, therefore its total elimination was the only option. Another argument in favour of such a procedure was the fact that the infection was caused by intestinal bacteria (E. faecalis), as a full recovery in such cases can only be achieved through the complete elimination of artificial material (Seabroock,1990). Because of the severe symptoms of ischemia of the lower limbs prior to the primary surgery, simultaneous vascular reconstruction was necessary.

Revascularization at the site of infection, because of the high risk of re-infection of the new bypass, requires the implantation of biological material (autologous vessels or cadaver homograft) or synthetic grafts more resistant to infections (antibiotic-soaked or impregnated with silver). The latter are mainly used in the case of deficiency of the biological material. In fact, results obtained in recent years have shown in fact no antimicrobial activity of prostheses impregnated with silver and a high risk of their recurrent infection (Batt et al., 2008). On the other hand, experimentally proven effectiveness of grafts coated with antibiotics to prevent intraoperative infections does not resolve doubts about their long-term antibacterial effect, resulting from the gradual progressive decrease of local concentration of antibiotics and increasing antibiotic resistance (Lew and Moore, 2011). The use of homografts, with significantly higher resistance to reinfections, is inextricably linked to their limited supply (requiring access to a tissue bank), gradual degeneration (due to the immunological rejection reactions), and retrograde changes (which are a consequence of conservation by freezing), resulting in true aneurysms, spontaneous rupture of the graft wall, anastomotic bleeding and thrombotic complications, as well as the risk of transmission of viral diseases (Verhelst et al., 2000). Admittedly, the use of immunosuppression prolongs the proper functioning of allogeneic grafts, but requires continuous application (Pupka et al., 2004). A way to circumvent these problems may be the use of autologous femoral veins, which provide all of the benefits associated with the use of biological material (the least vulnerability to superinfection, the ability to fully grow into a focus of infection) in the simultaneous absence of risks specific to the allografts (Dirven et al., 2015). The disadvantages of this method include extended operation time and unfortunately common disproportion between the available resource of veins and the length of the prosthesis, which they ought to replace. The first one loses its importance due to improvements in surgical technique (BudtzLilly et al., 2014), however to overcome the other, an additional source of material for the reconstruction of the vascular bed is required (with properties as close as possible to transplant tissue). The authors decided to use, as a supplement of the patient's femoral veins, a composite biosynthetic vascular graft Omniflow II. Its complex structure is created by a polyester mesh endoskeleton, which is the endurance element providing mechanical resistance and which is a kind of scaffolding for a biological component and the covering coating of ovine collagen allowing overgrowth of the graft wall by the vessels and tissues of the recipient. 
Omniflow's II high ability of incorporation into the host tissue has been known for over 20 years (Werkmeister et al., 1995), which makes it an alternative to autologous material during the preparation of the vascular graft (Dünschede et al., 2015) or arteriovenous dialysis access (Palumbo et al., 2009). Until recently, due to the lack of manufacturer's recommendations for Omniflow II implantation within the foci of infection, its use in the treatment of vascular graft infections was avoided. In 2012, Töpel et al., presented the first positive results of the use of these prostheses (in two cases combined with saphenous vein) for replacement of an infected graft located below the inguinal ligament (Töpel et al., 2012). Other authors have arrived at similar findings (Fellmer et al., 2014; Wiltberger et al., 2014).

In 2014, Neufang took advantage of these bioprostheses, combined with autologous superficial veins, to create vascular spans allowing to bypass long-segment obstructions of the arteries of the lower limbs. In the described cases, Omniflows II were the proximal parts of those spans (Neufang et al., 2014). So far the only work devoted to reconstructions using Omniflow II grafts in the aortofemoral segment was published in 2015 (Krasznai et al., 2015). Most authors highly evaluated the bioprosthetic Omniflow II in terms of their resistance to reinfection and degenerative changes. Despite the high resistance to infection, the authors limited the scope of the use of the mentioned prosthesis to the proximal section of aortobifemoral graft only. This was due to the presence of purulent fistulas in both groins, which were the locations of distal anastomoses. The authors concluded that the use of a bypass comprising even small amounts of synthetic material, in such conditions can cause a reinfection. The applied method of anatomical revascularization, using a combination of prosthesis Omniflow II with bilateral venous grafts allows the rational use of the existing stock of autologous vessels, as well as the simultaneous use of biosynthetic material in areas of reduced risk of infection. At the same time, however, it should be noticed that the combined character of the grafts carries the risk of potential stenoses in multiple anastomoses and requires regular clinical and radiological controls to provide their early identification.

\section{Literature}

Bandyk D.F., T.M. Bergamini, E.V. Kinney, G.R. Seabrook and J.B. Towne. 1991. In situ replacement of vascular prostheses infected by bacterial biofilms. J. Vasc. Surg. 13: 575-583.

Batt M., E. Jean-Baptiste, S. O'Connor, P.J. Bouillanne, P. Haudebourg, R. Hassen-Khodja, S. Declemy and R. Farhad. 2008. In situ revascularisation for patients with aortic graft infection: a single centre experience with silver coated polyester grafts. Eur. J. Vasc. Endovasc. Surg. 362: 182-188.

Bronk M. and A. Samet. 2008. Hospital enterococcal bacteremias. Post. Mikrobiol. 47: 339-344.

Budtz-Lilly J., N. Eldrup, S. Vammen and J. Laustsen. 2014. Femoral vein repair of arterial infections: technical tips to reduce procedure time. Vasc. Endovascular Surg. 48: 367-371.

Dirven M., M.F. van der Jagt, W.B. Barendregt and D. van der Vliet. 2015. The efficacy of autologous femoropopliteal vein reconstruction for primary aortic and aortic graft infection. Ann. Vasc. Surg. 29: 1188-1195.

Dünschede F., J. Stabrauskaite, G. Weisser, C. Espinola-Klein, B. Dorweiler and C.F. Vahl. 2015. Crural bypass for critical lower limb ischemia with Omniflow II prosthesis. Thorac. Cardiovasc. Surg. 64: 311-315.

Fellmer P.T., G. Wiltberger, H.M. Tautenhahn, I. Matia, F. Krenzien and S. Jonas. 2014. Early results after peripheral vascular replacement with biosynthetic collagen prosthesis in cases of graft infection (in German). Zentralbl Chir. 139: 546-551.

Krasznai A.G., M. Snoeijs, M.P. Siroen, T. Sigterman, A. Korsten, F.L. Moll and L.H. Bouwman. 2015. Treatment of aortic graft infection by in situ reconstruction with Omniflow II biosynthetic prosthesis. Vascular. DOI: 10.1177/1708538115621195.

Lew W. and W. Moore. 2011. Antibiotic-impregnated grafts for aortic reconstruction. Semin. Vasc. Surg. 244: 211-219.

Neufang A., B. Dorweiler, C. Espinola-Klein, S. Savvidis, M. Doemland, S. Schotten and C.F. Vahl. 2014. Outcomes of complex femorodistal sequential autologous vein and biologic prosthesis composite bypass grafts. J. Vasc. Surg. 60: 1543-1553.

Palumbo R., P. Niscola, S. Calabria, S. Fierimonte, M. Bevilacqua L. Scaramucci, B. Tolu, P. de Fabritiis and F. Bondanini. 2009. Long-term favorable results by arteriovenous graft with Omniflow II prosthesis for hemodialysis. Nephron. Clin. Pract. 1132: 76-80.

Pupka A., B. Dolińska-Krajewska, S. Pawłowski and P. Szyber. 2004. Immunosuppression in the transplantation of the fresh arterial homograft - case report. Adv. Clin. Exp. Med. 13: 371-374.

Samson R.H., F.J. Veith, G.S. Janko, S.K. Gupta and L.A. Scher. 1988. A modified classification and approach to the management of infections involving peripheral arterial prosthetic grafts. J. Vasc. Surg. 8: 147-53.

Seabroock G.R. 1990. Pathobiology of graft infections. Sem. Vasc. Surg. 3: 81-88.

Swain T.W., K.D. Calligaro and M.D. Dougherty. 2004. Management of infected aortic prosthetic grafts. Vasc. Endovascular Surg. 38: 75-82.

Töpel I., T. Betz, C. Uhl, M. Wiesner, S. Bröckner and M. Steinbauer. 2012. Use of biosynthetic prosthesis (Omniflow II) to replace infected infrainguinal prosthetic grafts - first results. Vasa. 413: 215-220.

Verhelst R., V. Lacroix and H. Vraux. 2000. Use of cryopreserved arterial homografts for mamagement of infected prosthetic grafts: a multicentric study. Ann. Vasc. Surg. 14: 602-607.

Werkmeister J.A., J.F. White, G.A. Edwards and J.A. Ramshaw. 1995. Early performance appraisal of the Omniflow II vascular prosthesis as an indicator of long-term function. J. Long Term. Eff. Med. Implants. 5: 1-10.

Wiltberger G., I. Matia, M. Schmelzle, F. Krenzien, H.M. Hau, B. Freitas, S. Jonas and P.T. Fellmer. 2014. Mid- and long-term results after replacement of infected peripheral vascular prosthetic grafts with biosynthetic collagen prosthesis. J. Cardiovasc. Surg. 55: 693-698. 